\title{
An Experimental Study of the Limitations of Mobile Haptic Interfaces
}

\author{
F. Barbagli ${ }^{1,2}$, A. Formaglio ${ }^{1}$, M. Franzini ${ }^{1}$, A. Giannitrapani ${ }^{1}$, and \\ D. Prattichizzo ${ }^{1}$ \\ (1) Dipartimento di Ingegneria dell'Informazione, University of Siena \\ (2) Stanford Robotics Lab, Stanford University \\ [barbagli, formaglio, anto, franzini, prattichizzo] @ dii.unisi.it
}

\begin{abstract}
This paper presents various procedures that can be used in order to numerically evaluate what the maximum $Z$-width that can be rendered by a mobile haptic interface will be given few parameters that characterize the haptic device and the mobile platform that make up such interface. Such procedures are applied to the case of two different mobile haptic interfaces. Results are encouraging, even though limitations to the proposed procedure exist.
\end{abstract}

\section{Introduction}

The workspace of haptic interfaces varies largely on their design and usage, ranging from few planar centimeters of the Pantograph [2] to several cube meters of the Scaleable Spidar device [3]. Most haptic devices, however, share two main traits: they are grounded and they have limited workspace. While this is not a problem in many applications, it can become one in cases where users need to interact with large virtual environments while navigating inside of them.

A possible solutions for this problem is to use locomotion interfaces, i.e. treadmilllike interfaces that simulate some of the inertial feedback that a user would experience while navigating through a large virtual environment [5]. Another possible approach is to create haptic interfaces featuring unlimited workspace by combining mobile robots and standard grounded force-feedback devices. This type of interface, which in part resembles the cobot [4], was introduced by Nitzsche et al [1] and is referred to as mobile haptic interface. In this paper we focus our attention on the latter type of interfaces (see Fig. 1 for two examples of mobile haptic interfaces).

Haptic interfaces are often described as mechanical impedance transducers. Mobile haptic interfaces are no exceptions, and are thus required to render impedances ranging from zero, simulating movement in free space, to a maximum value $Z_{\max }$ that depends on a multitude of factors [6]. The $Z$-width characterizing a mobile haptic interface depends on factors characterizing its two main constitutive subsystems.

The main contribution of this paper is to attempt to provide some useful guidelines on how to select the right combination of a desktop haptic device and a mobile platform in order to design a mobile haptic interface that will have a certain desired $Z$-width. More specifically we will focus our attention on how to correctly simulate 

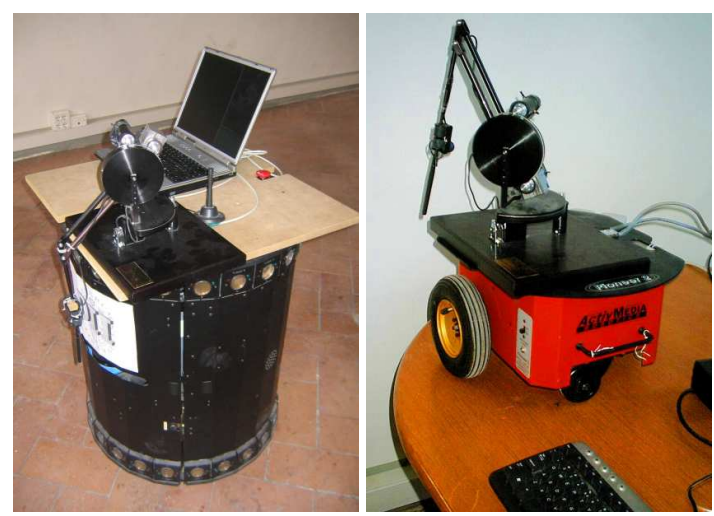

Fig. 1. Two mobile haptic interfaces: a general purpose interface based on the holonomic Nomad mobile robot and a Phantom Premium 1.5; a more limited interface based on a non-holonomic Pioneer mobile robot and a Phantom Premium 1.5

free-space movement, i.e. $Z_{\min } \simeq 0$. In order to do so we will first introduce a simplified model of a mobile haptic interface (Section 2); we will then use such model to analyze the response of a mobile haptic interface to a given set of input signals that most closely resemble those of the users (Section 3); finally we will compare such results to a set of experimental results obtained on different mobile haptic interfaces (Section 4). While the simplicity of the proposed model may lead to some error in our analysis, its usage allow the results obtained to be easily interpretable.

\section{Modelling mobile haptic interfaces}

Mobile haptic interfaces (MHI) are made up of two main components: a mobile platform (MP), such as the holonomic Nomad or the non-holonomic Pioneer mobile robots in Fig. 1; an impedance-type haptic device (HD), such as the Phantom device in Fig. 1, grounded to the mobile platform. The former robots typically feature high inertia and are normally position controlled. The latter normally feature low inertias and high levels of transparency and are normally controlled in force.

In order to transparently render any impedance inside an unlimited environment we propose a simple control algorithm, which mimics the one proposed by Nitzsche et al. in [1]. The basic idea is that the MP should be position controlled to track the movements of the operator, thus allowing the HD to always be in the center of its workspace. This has two main advantages: it allows the HD to render forces on the operator in a configuration of maximum structural stiffness; and it allows the HD to never reach its workspace limits, a situation in which spurious forces would be felt by the operator thus destroying the overall sense of transparency. On the other side, forces are rendered by the HD using standard constrained based methods such as the proxy algorithm [7].

Let $\Sigma_{W}$ be the base reference frame which is attached to the world and $\Sigma_{M}$ the reference frame attached to the mobile platform base. 


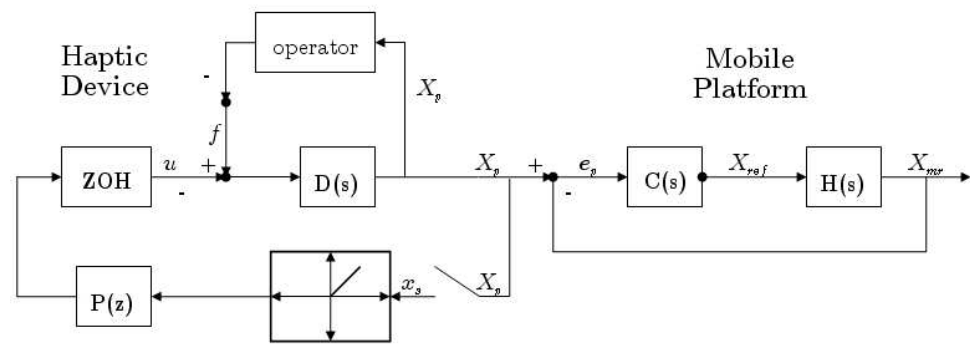

Fig. 2. Control scheme for a mobile haptic interface: the left side of the picture represents the interaction force rendering algorithm while the right side represents the position control algorithms for the mobile platform.

In order to control the position of the MP we propose a simple PD controller as depicted in Fig. 2 (right side), where $X_{p}$ represents the position of the HD end effector with respect to $\Sigma_{W}, X_{m r}$ the position of the MP with respect to $\Sigma_{W}, e_{p}$ is the position of the HD end effector with respect to $\Sigma_{M}$ (as well as the tracking error for the mobile platform), $X_{r e f}(s)=C(s) e_{p}(s)$ is the position commanded to the MP, $C(s)$ is the transfer function of the MP control algorithm, and $H(s)$ is the transfer function representing the MP.

In order to control the interaction forces between user and virtual environments we use a classic scheme that was introduced by Colgate in [6], see Fig. 2 (left side) where $X_{p}$ has been defined above, $D(s)$ is the haptic device transfer function, $P(z)$ is the discrete time transfer function of the virtual object impedance, and $\mathrm{ZOH}$ is a zero holder hold. Note that while this simplified model is normally used to represent virtual walls it can be generalized to more complex cases by adding a collision detection block.

The MP can be modelled as a mass-damping system subject to actuator forces, which are modelled using a spring. Thus in the case of the MP we have $F_{m r}(s)=$ $K_{m} X_{r e f}(s)-B_{m} s X_{m r}(s)$ yielding transfer function

$$
H(s)=\frac{K_{m}}{s\left(M_{m} s+B_{m}\right)}=\frac{X_{m w}}{X_{r e f}} .
$$

While this is far from being an exact dynamical model of a mobile robot and cannot account for non-linear effects that are present in reality, it has the advantage of being simple and of being characterized by a small set of parameters that can easily be interpreted and identified. Both MPs that were employed during the experimental phase have been modelled using this approach.

A PD controller is chosen in order for the platform to track the position of the user with respect to the world, i.e. to bring $e_{p}$ to zero. Thus $C(s)=s D_{m}+P_{m}$.

The HD can also be modelled as a mass-damper system such that

$$
D(s)=\frac{1}{s\left(M_{h} s+B_{h}\right)}
$$


and similarly the impedance model of a virtual object can be chosen as a discrete spring-damper system, yielding $P(z)=D_{h} \frac{z-1}{z T}+P_{h}$ where $T$ is the haptic servoloop period.

\section{Performance of a mobile haptic interface}

The $Z$-width of a MHI depends on how MP and HD are controlled. Standard $\mathrm{HD}$ are typically designed in order to have low reflected inertias and friction, i.e. $Z_{\min } \simeq 0$, while $Z_{\max }$ normally depends on the servo rate at which the device can be commanded [6]. The addition of a MP, whose inertia is usually fairly large and whose dynamics are normally slower than those of the HD, has little implication on $Z_{\max }$ but major implications on $Z_{\min }$. When a user is slowly examining a virtual object by touching it with the HD the MP basically does not move, and thus $Z_{\max }$ is determined by the HD alone. When a user moves in free space, on the other side, the MP may lag behind the HD, leading the user to feel spurious forces due to the HD workspace limitations, i.e. experiencing a $Z_{\min }>0$. In such case the control scheme on Fig. 2 ceases to be valid and $u$ is determined by the contact with the workspace limits of the HD.

The goal of this section is to analytically determine which free space motions of the user will result in a correct impedance being rendered by the MHI. Thus we seek to determine $X_{p}$ such that $e_{p}<X_{w s}$ with $X_{w s}$ workspace limits of the HD. Such analysis will focus on three main types of inputs that closely resemble an operator's movements: step input, ramp input, and sinusoidal input. It is important to note that our analysis only applies to steady-state behavior of the system. This does not cover all possible scenarios, since during transients the HD could reach its workspace limits, and thus the analytical results proposed in the following sections must be considered as rough indicators of performance.

\subsection{Step displacement}

What is the maximum step displacement $X_{p}(t)=A_{g},(t \geq 0)$, that can be correctly rendered by the MHI? Let us consider an ideal step signal of amplitude $A_{g}$. At time $t=0^{+}$we have

$$
X_{m r}\left(0^{+}\right)=0 \text { and } X_{p}\left(0^{+}\right)=A_{g}
$$

i.e. while the system output is still at zero the input has jumped to $A_{g}$. In this case $e_{p}\left(0^{+}\right)=A_{g}$, i.e. the haptic device is at a $A_{g}$ distance from the center of its workspace, and thus it is necessary ${ }^{1}$ that the maximum amplitude of a step position signal applied to a MHI is such that $A_{g}<X_{w s}$.

\subsection{Ramp displacement}

What is the maximum ramp displacement $X_{p}=V_{R} t$ that can be correctly rendered by the MHI? Let us consider an ideal ramp $X_{p}=V_{R} t$. The MP model of Fig. 2

\footnotetext{
${ }^{1}$ but not suffi cient due to the lack of knowledge of what happens during transients
} 
follows such input with a finite steady-state error, since $H(s)$ has one pole in the origin. Such error is given by

$$
e_{p}(\infty)=\lim _{s \rightarrow 0}\left(\frac{1}{s C(s) H^{\prime}(s) \frac{1}{s}}\right) V_{R}=\frac{V_{R}}{K_{v}}
$$

where $H(s)=H^{\prime}(s) / s$. Note however that the maximum error between HD and MP positions $e_{\max }$ can be reached during the transient and can be found using

$$
e_{p o} \triangleq \frac{e_{\max }-e_{p}(\infty)}{e_{p}(\infty)}
$$

where $e_{p o}$ does not depend on the slope of the ramp but only on $\mathrm{H}(\mathrm{s})$ and $\mathrm{C}(\mathrm{s})$. Given that $e_{\max }$ is proportional to $V_{R}$, since $e_{\max }=\left(1+e_{p o}\right) \frac{V_{R}}{K_{v}}$, by experimentally determining the value of $e_{p o}$ for a given MP, we can then compute $e_{\max }$. In order for $e_{\max }<X_{w s}$ to be true, when applying $X_{p}(t)=V_{R} t, V_{R}$ must be such that

$$
V_{R}<\frac{X_{w s} K_{v}}{\left(1+e_{p o}\right)}
$$

\subsection{Sinusoidal displacement}

What is the maximum amplitude/frequency of $X_{p}=A_{s} \sin (\omega t)$ that can be correctly rendered by the MHI? Referring to Fig. 2 it is possible to compute $G(s)$ such that $e_{p}(s)=G(s) X_{p}(s)$. Given the linearity of the overall system we have

$$
e_{p}=A_{s}\|G(j \omega)\| \sin (\omega t+\angle(G(j \omega)))
$$

and thus in order for $e_{p} \in\left(-X_{w s} ; X_{w s}\right)$, inequality $A_{s}\|G(j \omega)\|<X_{w s}$ must hold. Since $G(s)$ has high-pass filter behavior, higher-frequency sinusoids must have lower amplitude in order for the MHI to track them and viceversa.

In other terms it is always possible to compute a region of the $\left(\omega, A_{s}\right)$ plane, $\mathcal{I}=\left\{\left(\omega, A_{s}\right) /\left(\omega, A_{s}\right): A_{s}\|G(j \omega)\|<X_{w s}\right\}$, that represents the sinusoidal inputs that can be correctly rendered by a MHI. Moreover curve $\gamma: A_{s}\|G(j \omega)\|=$ $X_{w s}$ representing the border between $\mathcal{I}$ and the rest of the $\left(\omega, A_{s}\right)$ plane can be numerically computed.

\section{Experimental results}

In order to validate the theoretical results presented in Section 3, several experimental tests have been carried out, using the Phantom Premium 1.5 haptic interface and the mobile platforms Pioneer2 DX and Nomad XR4000. The latter is a fully holonomic robot, featuring high levels of inertia, whereas the former is an agile differential drive (non-holonomic) robot.

A preliminary set of experiments has been conducted to identify the model parameters of each robot. For this purpose, several sets of input-output data $\left\{X_{r e f}, X_{m r}\right\}$, 
corresponding to different kinds of input signals (square waves, ramps, sinusoids) have been collected, and the values of the parameters $K_{m}, M_{m}, B_{m}$ characterizing the transfer function $H(s)$ (see Section 2) have been tuned, by comparing the actual and simulated outputs. Once the robot models were available, a suitable PD controller has been designed to guarantee closed-loop stability and to achieve satisfactory tracking performance.

Two experimental setups have been considered, combining the HD with each MP. All tests have been performed along a single degree of freedom and for each experimental trial, the actual tracking error has been compared to the one predicted by the corresponding model. As pointed out in Section 2, the tracking error $e_{p}$ corresponds to the displacement of the end-effector w.r.t. the center of its workspace, thus being directly available from the readings of the haptic interface encoders.

In order to verify the ability of the models to predict whether a given input $X_{p}$, belonging to one of the signal classes considered in Section 3, would cause the endeffector to reach the limit of its workspace, several signals within each class have been applied to the haptic device end-effector. During all the experimental trials, it was supposed that the maximum desired displacement of the end-effector from the center of its workspace was $X_{w s}=150 \mathrm{~mm}$. In Fig. 4 and 5, the results of typical experimental tests, involving the Pioneer2 DX and the Nomad XR4000 MP, respectively, are shown.

\subsection{Step displacement}

In a first set of experiments, step displacements $X_{p}(t)=A_{g},(t \geq 0)$, with different amplitudes, have been used as reference signals. The ideal step has been generated by fixing the haptic device's end-effector to a given position $A_{g}$ to the side of the center of the workspace, before the robot starts the tracking. As correctly predicted by the models, the results obtained with both MHI configurations confirmed that as long as the amplitude of the Step displacements is inside the workspace limit, the end-effector will never reach its maximum extension (see Fig. 4(a) and 5(a)).

\subsection{Ramp displacement}

Secondly, Ramp displacements $X_{p}(t)=V_{R} t$ have been considered. In order to move the haptic device's end-effector with a constant velocity $V_{R}$, a second mobile robot was used. Thus, while the MHI stood still, another mobile robot was accelerated in order to reach a desired velocity $V_{R}$ (see Fig. 3) and then hooked up to the MHI end-effector (through a velcro connection), thus exciting the MHI with the desired reference signal. With this kind of inputs, the behavior of the two MHI configurations is significantly different. As far as the Pioneer platform is concerned, it turns out that the maximum error is reached during the transient (see Fig. 4(b)-4(c)), whereas the MHI employing the Nomad robot reaches its maximum tracking error at the steady state (see Fig. 5(b)-5(c)). This is due to the different controller parameters, which make the Pioneer platform exhibit a remarkable overshoot. The maximum tracking error of the Nomad MHI is almost proportional to the input velocity, as predicted by 
the theoretical analysis, contrariwise to the Pioneer behavior, manifesting nonlinear phenomena.
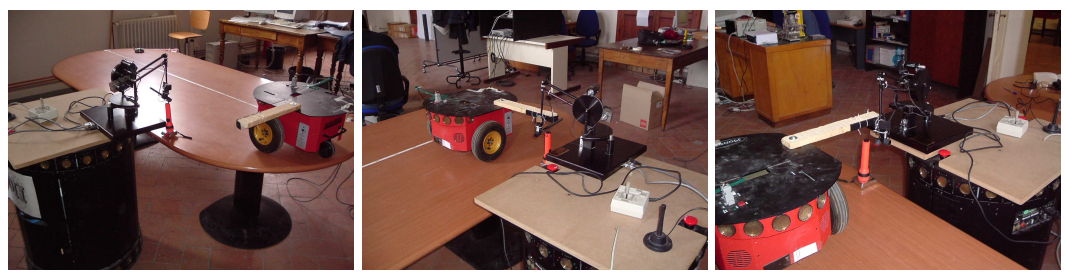

Fig. 3. A mobile robot is used to excite the MHI with a ramp signal: (left) the mobile robot is accelerated to reach $V_{R}$ velocity; (middle) the mobile robot comes into contact with the MHI end effector; (right) the MHI end effector starts moving.

\subsection{Sinusoidal displacement}

The last set of experiments have been performed with sinusoidal inputs $X_{p}(t)=$ $A_{s} \sin (\omega t)$, featuring different amplitudes $A_{s}$ and frequencies $\omega$. Such reference signals have been generated by a human operator who, with the aid of periodic acoustic and visual stimuli, moved the haptic device's end-effector sideways, approximately describing a time dependent sinusoid.

The experimental results obtained with sinusoidal inputs support the intuition that, in order to confine the tracking error within the workspace limits, the maximum admissible amplitude is roughly inversely proportional to the sinusoid frequency. In this case, the maximum tracking error predicted by the Pioneer MHI is significantly bigger than the actual one, thus resulting in more conservative constraints on the admissible sinusoidal inputs (see Fig. 4(d)-4(e)). This means that a more extensive model identification phase is needed. On the contrary, the maximum predicted and actual error for the Nomad MHI are very close (5(d)-5(e)). Due to the inaccuracy of the man-made sinusoidal references as well as nonlinear phenomena, the actual tracking error may exhibit trends and/or non-zero mean.

In summary, the performed experimental phase confirmed the viability of the presented theoretical analysis, showing that it may serve as a useful tool for the evaluation of MHI's performance limitations. Despite the difficulty to accurately reproduce the time evolution of the tracking error, the procedures proposed in this paper were able to correctly predict the outreach of the HD workspace.

It is worth noticing that, due to the simple structure of the chosen robot model, the resulting theoretical limitations on the trackable input signals may be somewhat conservative. Of course, a noticeable improvement would be achieved by adopting more sophisticated robot motion models and carrying out more extensive parameter identification campaigns.

We conclude this section pointing out some technical problems encountered during the experimental phase. 


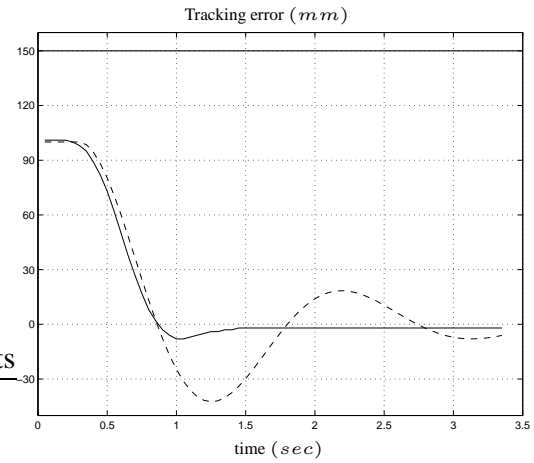

(a) Step input: $X_{p}(t)=100$

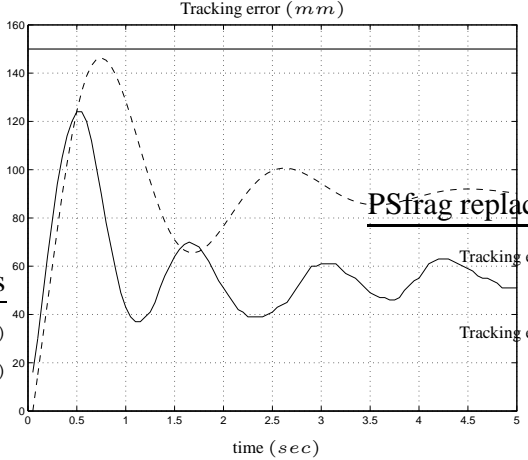

(b) Ramp input: $X_{p}(t)=300 t$

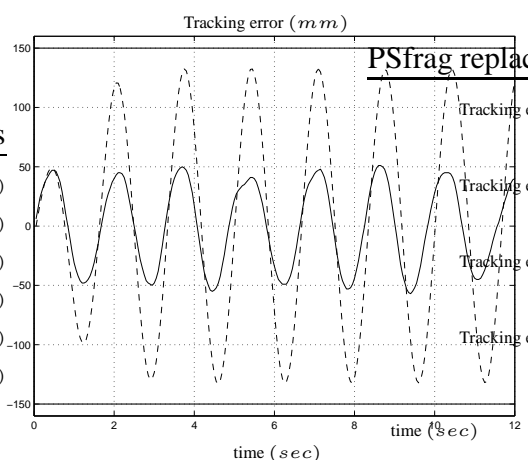

(d) Sinusoidal input: $X_{p}(t)=50 \sin (2 \pi 0.6 t)$

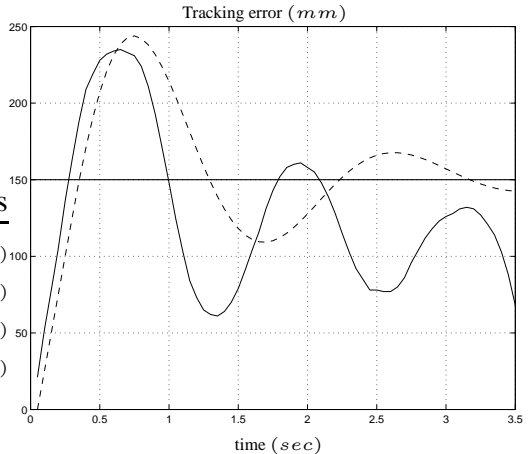

(c) Ramp input: $X_{p}(t)=500 t$

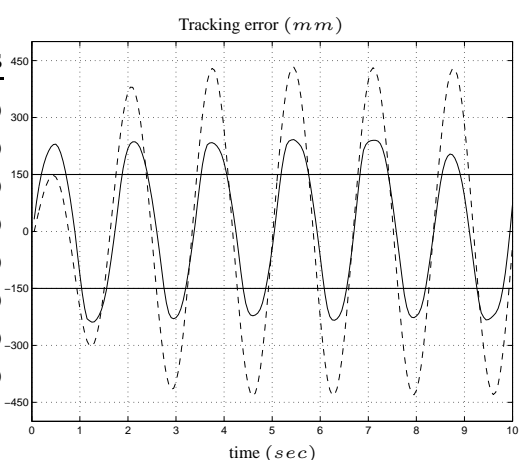

(e) Sinusoidal input: $X_{p}(t)=150 \sin (2 \pi 0.6 t)$

Fig. 4. Experimental results with the Pioneer2 DX mobile platform and different input signals: actual (solid line) and predicted (dashed line) tracking error. Thick solid lines represent the desired maximum error, due to workspace limits. 


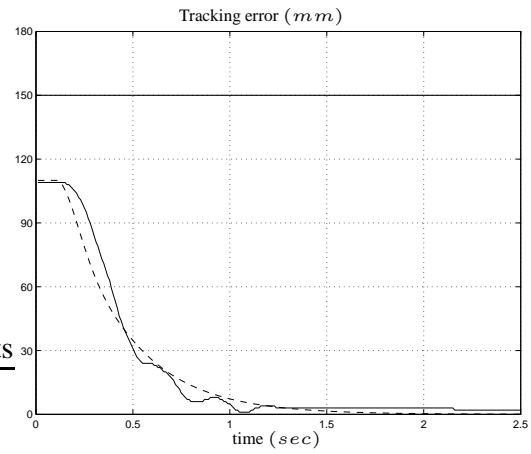

(a) Step input: $X_{p}(t)=110$

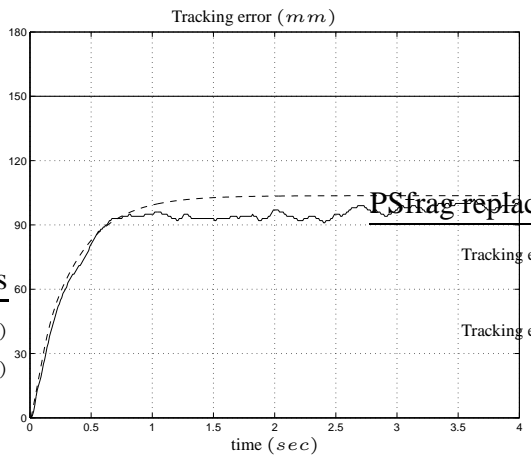

(b) Ramp input: $X_{p}(t)=300 t$

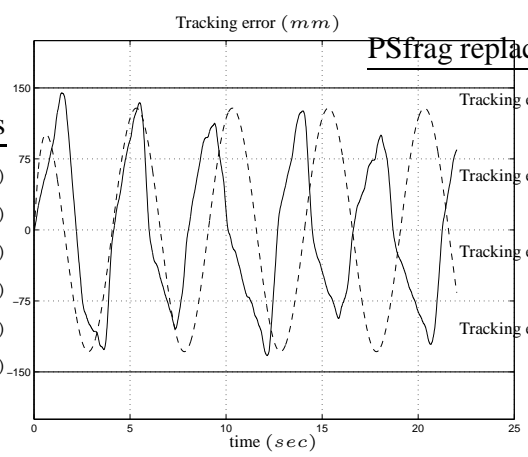

(d) Sinusoidal input: $X_{p}(t)=250 \sin (2 \pi 0.2 t)$

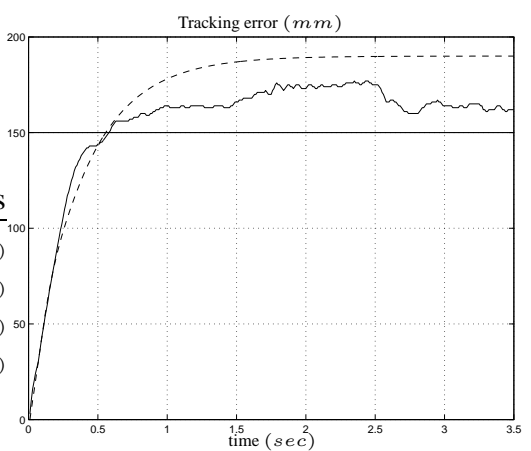

(c) Ramp input: $X_{p}(t)=500 t$

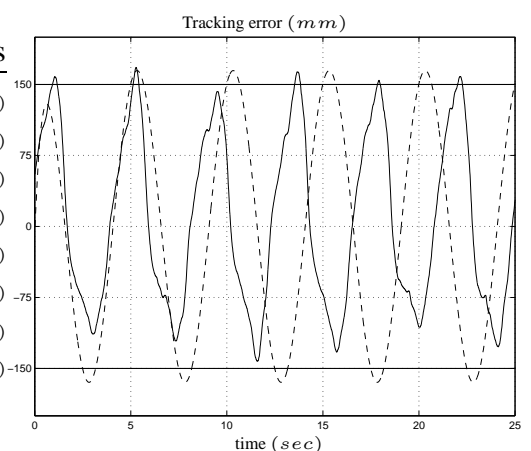

(e) Sinusoidal input: $X_{p}(t)=300 \sin (2 \pi 0.2 t)$

Fig. 5. Experimental results with the Nomad XR4000 mobile platform and different input signals: actual (solid line) and predicted (dashed line) tracking error. Thick solid lines represent the desired maximum error, due to workspace limits. 
- The generation of accurate sinusoidal reference signals by means of a MP was not possible due to spurious movements during the inversions of the motion direction. In these situations the Nomad platform suffers from the problem of sudden wheel steering, while the requested acceleration makes the Pioneer robot slip. The same undesirable phenomena are experienced when the MHI tries to track a man-made sinusoidal input.

- The range of testable reference signals is severely limited by a number of factors. First, the movements of the robots are constrained by the length of the Phantom Premium 1.5 cables (roughly 2 meters). Secondly, for safety reasons, special caution must be used when using the Nomad platform, which basically results in limiting the maximum admissible speed of the reference signals. Finally, special care should be devoted for preventing the controller to excite un-modelled dynamics or inevitable nonlinearities (e.g., actuator saturations).

- The communication between the haptic device and the MP occurs via serial link (Pioneer robot) or Ethernet connection (Nomad robot). This introduces significant delays and degrades the tracking performance.

\section{Conclusion and future work}

This paper presents various procedures that can be used to pre-evaluate if the minimum impedance that can be rendered by a MHI can be made equal to zero. The proposed procedures are shown to match the real behavior of two MHI with different features. However such procedures have limitations, mainly due to the fact that they can only account for steady state behavior and that MHIs are modelled as linear systems. Thus the proposed procedures should only be considered as qualitative indicators of the likely performance of an MHI, and not as exact ones.

\section{References}

1. N. Nitzsche, U.D. Hanebeck, and G. Schmidt "Desgin Issues of Mobile haptic Interfaces", Journal of Robotic Systems, vol. 20:9, pp. 549-556, 2003.

2. C. Ramstein and V. Hayward, "The Pantograph: A Large Workspace Haptic Device For A Multi-Modal Human-Computer Interaction”, CHI'94, Conference on Human Factors in Computing Systems ACM/SIGCHI Companion-4/94, pp. 57-58, 1994.

3. L. Bouguila, M. Ishii and M. Sato, "Scaleable SPIDAR: A Haptic Interface For HumanScale Virtual Environments", Haptic Human-Computer Interaction, pp. 182-193, 2000.

4. M. Peshkin, J. E. Colgate, W. Wannasuphoprasit, C. Moore, B. Gillespie and P. Akella, "Cobot Architecture", IEEE Tr. on Robotics and Automation, 17(4):377-390, 2001.

5. J. M. Hollerbach et al., "Simulating side slopes on locomotion interfaces using torso forces," Haptic Symposium, pp. 91-98, March 22-23, 2003.

6. J. E. Colgate and J. M. Brown, "Factors Affecting the Z-Width of a Haptic Display", in Proceedings of the IEEE International Conference on Robotics \& Automation, pp. 3205-10, San Diego, CA, May 1994.

7. D. C. Ruspini, and K. Kolarov and O. Khatib, "The Haptic Display of Complex Graphical Environments”, Siggraph97, pp. 345-352, 1997. 